\title{
LA ESCOLARIZACIÓN DE LOS NIÑOS GITANOS EN EL PAÍS VALENCIANO
}

\section{JOSÉ CABANES HERNÁNDEZ}

Sociólogo. Servicios Sociales Municipales de Villena (Alicante). Monitor de minorías étnicas.

\section{INTRODUCCIÓN}

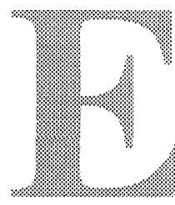

sta exposición es fruto de un estudio, no finalizado, que se realizó entre septiembre de 1996 y diciembre de 1997. El estudio fue encargado por la Consellería de Bienestar Social, por medio de una única beca de investigación, y en él participaron más tarde cerca de un centenar de voluntarias/os (estudiantes del último curso de Trabajo Social, y de Sociología, profesoras/es de la Escuela de Trabajo Social de Alicante, trabajadoras sociales, monitores de seguimiento escolar, etc.), sin cuyo trabajo hubiese resultado imposible llevar a cabo un estudio con tan amplia acotación geográfica.

El principal objetivo del estudio era el tener un acercamiento muy genérico a la situación gitana de todo el país valenciano (de cada una de sus localidades), y en diversas cuestiones (origen, barrios de residencia, tipo de viviendas, número aproximado de habitantes gitanos, situación escolar, migraciones y relaciones interétnicas), lo que suponía, de entrada, el no poder profundizar en ninguna de las cuestiones ni de las localidades.

En el apartado escolar, la información se ha obtenido por medio de entrevistas a los directores, jefes de estudios o claustros (según colegios) de 230 centros escolares de los distintos municipios valencianos, con un total de 5.000 niños y niñas gitanas, y complementada en las entrevistas a los centros sociales y familias gitanas (se han entrevistado a todos los equipos de Servicios Sociales Municipales, así como a distintas familias gitanas y asociaciones en los municipios).

\section{PROCESO HISTORICO, MIGRACIONES Y DISTRIBUCIÓN ACTUAL.}

El País Valenciano ha contado históricamente con un importante número de familias gitanas, debido especialmente a su riqueza agrícola, que permitía realizar trabajos como jornaleros en el campo y que también potenciaba un importante mercado de compraventa de caballerías. 
Con la industrialización de la agricultura en los años 60, muchas familias gitanas en todo el Estado pierden su principal ocupación, la compraventa de caballerías, y se produce un importante flujo migratorio de familias gitanas (década de los 60 y primera mitad de los 70) de Andalucía (principalmente Jaén y Granada) y Castilla la Mancha, mayoritariamente, aunque también de otras muchas regiones. Estas migraciones buscan principalmente la posibilidad de encontrar trabajo como temporeros agrícolas, y en muchas localidades suplen la mano de obra abandonada por los trabajadores "payos" que habían pasado a trabajar en el sector industrial. Del mismo modo, muchos pequeños pueblos (especialmente de montaña) ven desaparecer a sus vecinos gitanos en busca de mejor fortuna en localidades más agrícolas (actualmente, hay unas 356 localidades donde no reside ninguna familia gitana).

Entre la segunda mitad de los años 70 y la primera de los 80 se da el fenómeno de la macro construcción de bloques viviendas sociales en distintos puntos de la Comunidad Valenciana, especialmente en aquellos donde ya existían problemas de vivienda precaria. Este nuevo fenómeno, en un principio dirigido a familias chabolistas, atrajo un nuevo flujo migratorio de familias que residían en otras localidades, en ocasiones de provincias muy alejadas, en busca de la oportunidad de acceder a una vivienda barata. Es el periodo en el que se consolidan los numerosos barrios ghetto que se distribuyen en las principales ciudades, y con ellos los principales colegios donde acabaran por concentrarse casi exclusivamente los niños gitanos. Aunque no los únicos colegios donde esto sucede.

Ya en los principios de los años 90 ha empezado a darse un proceso migratorio diferente, el cual es en dirección inversa a los anteriores, o sea, ciudad-campo. Se trata, por lo general, de familias que tratan de alejarse de las grandes concentraciones de población gitana (en especial por miedo a que sus hijos crezcan cerca del problema de la droga) y que buscan localidades cercanas a sus zonas de venta (si son vendedores ambulantes) o con recursos laborales (especialmente en el sector agrícola). Al mismo tiempo, se han sucedido nuevas migraciones desde Andalucía, que se instalan en localidades con trabajos agrícolas (en la mayoría de los casos sin población gitana anterior o con gitanos familiares de éstas) y que combinan su estancia en el municipio con las peonadas agrícolas en sus localidades de origen, a las que acuden durante unos meses al año. Por lo general, y con significativas excepciones de familias muy desestructuradas ya en su localidad de origen, estas nuevas migraciones están consiguiendo una buena integración sociolaboral y, paralelamente, los niños y niñas una mejor adaptación y resultados escolares. 
En la actualidad residen en el País Valenciano alrededor de 50.000 gitanos (estimación aproximada) en un total de 180 localidades (de las 536 existentes), de las cuales en más de la mitad residen menos de 100 personas gitanas, y sólo 8 municipios superan los 1.000 habitantes gitanos.

\section{III.DIFERENTES SITUACIONES SEGÚN LOCALIDADES.}

Como ya todos sabemos, la situación escolar no suele ser más que un reflejo de la situación social que se vive en la calle. En este sentido, creo que es interesante exponer las diferentes situaciones sociales en que los gitanos viven en el País Valenciano y como éstas les afectan.

Cuando comenzamos a realizar este estudio existía la tentación de limitarnos a estudiar las localidades que cuentan con un número muy importante de gitanos y que, en general, suelen ser famosas por la existencia de diversos problemas socioeconómicos. Sin embargo, se desechó esta idea porque, de alguna forma, ese tipo de estudios no nos están permitiendo ver que la realidad de la población gitana es mucho más diversa.

En la práctica totalidad de las localidades donde existe un alto número de población gitana suelen repetirse diferentes tipologías de barrios de residencia que llevan a la concentración espacial de la mayoría (cuando no de todas) las familias gitanas. Y que repercuten de forma importante en la situación escolar. La política de vivienda ha conllevado por activa y por pasiva la concentración de la población de menores recursos en los mismos barrios de estas ciudades y, entre esa población, principalmente, a los gitanos. Por activa, mediante la construcción de bloques de viviendas sociales en la periferia de la ciudad, o, en ocasiones, un poco más allá, en el extrarradio, condenándoles a una marginalidad social (están físicamente al margen de la sociedad) y económica. Por pasiva, mediante el mercado libre de vivienda, donde un piso o una casa cuesta una fortuna, y que relega a los estratos socioeconómicos más bajos a concentrarse en los barrios donde no quiere vivir ya nadie (especialmente cascos viejos muy deteriorados, situados en la ladera de alguna montaña o castillo, o en barrios que se sitúan en la periferia de la ciudad separados de ésta por una autovía o la vía férrea). Muchas de las familias que residen en estos barrios suelen padecer problemas de desestructuración, que son a la vez causa y efecto de las condiciones en que se han visto obligadas a vivir. Este tipo de habitat de concentración espacial conlleva una concentración también escolar, que al poco tiempo acaba por convertir al centro en un ghetto de donde huyen los alumnos no gitanos. (Creo que la situa- 
ción es bien conocida por todos/as ya que se repite en una infinidad de ciudades de todo el Estado). En estas ciudades las relaciones interétnicas (payos-gitanos) no suelen ser muy fluidas, y a los prejuicios y estereotipos existentes ya, sobre los gitanos en general, se suma la estigmatización de los barrios de residencia, lo que conlleva una situación en que los dos grupos étnicos se relacionan poco y mal.

En cualquier caso, suele haber en estas ciudades un número de familias, normalmente bastante pequeño, que ha conseguido instalarse en otros barrios, de forma más dispersa y que, en general, tienen una buena situación económica y social. Los hijos de estas familias suelen acceder a colegios con un bajo porcentaje de niños/as gitanos y, en general, están consiguiendo unos mejores resultados académicos (aunque en muchos de los casos el fracaso escolar siga siendo importante).

Por su parte, creo que es resaltable la situación que se da en un gran número de pueblos, más de la mitad, donde la población gitana no supera el centenar de personas. En muchos de estos pueblos la situación laboral y urbanística ha permitido una mejor situación de las familias gitanas, las cuales suelen dedicarse principalmente a realizar trabajos agrícolas (que es en muchos casos también el trabajo principal de la población no gitana). En estas localidades las familias gitanas suelen residir de forma dispersa en distintas calles del pueblo, lo que proporciona una mayor fluidez en las relaciones interétnicas y una mejor situación sociolaboral. Los niños, por su parte, se distribuyen en distintos colegios (cuando no, en el único colegio del municipio) y su situación escolar suele ser parecida a la de sus compañeros no gitanos, dependiendo de algunas variables añadidas (la familia a la que pertenece y la prioridad que le da a la escuela; la localidad donde residen y su actitud hacia los gitanos, etc.).

Evidentemente, esta situación de "normalidad" no se da en todas las pequeñas localidades, y se debe mencionar que muchas veces el hecho de que en un pueblo no resida ninguna familia gitana se debe al rechazo social que en ese municipio se tiene contra los gitanos. Por poner un ejemplo: cuando comencé con la investigación llamé a los ayuntamientos de los distintos municipios para establecer un primer contacto y conocer si residían familias gitanas. En un Ayuntamiento, la Sra. Alcaldesa, tras explicarle el objeto del estudio, me respondió; "en este pueblo, gracias a Dios, no vive ningún gitano".

A pesar de las múltiples excepciones, creo que en las localidades donde reside un número no muy alto de familias gitanas se está consiguiendo una aceptable situación socioeconómica y, paralelamente, también escolar. En este sentido me parece importante el tímido, pero significativo, flujo migratorio que en algunas comarcas se está dando de familias gitanas que emigran de las grandes ciudades con alta con- 
centración de población gitana, hacia municipios pequeños donde hasta ahora no había vecinos gitanos.

\section{IV.ESCOLARIZACIÓN ACTUAL}

En la actualidad, la práctica totalidad de los niños y niñas gitanas se encuentran escolarizados, aunque todavía existen algunos casos muy puntuales donde se constata la desescolarización de algunos niños, especialmente en barrios del extrarradio de las dos mayores ciudades (Valencia y Alicante), así como hijos de familias recién inmigradas que no habían escolarizado a sus hijos previamente, o que no los escolariza al llegar a la localidad, quizás por un planteamiento de que se trata sólo de una situación temporal (es muy frecuente cuando la inmigración responde a una situación de temporerismo agrícola que ahora trataremos).

En algunos barrios de la ciudad de Valencia los Servicios Sociales estiman en un $5 \%$ el número de niños gitanos desescolarizados. Sin embargo, y aunque la cifra es muy alta en comparación con la mayoría de las localidades donde el $100 \%$ están escolarizados, un estudio sociológico realizado en 1985 en la ciudad de Valencia estimaba en un $44 \%$ el número de niños desescolarizados. Aunque ese dato puede ser un poco exagerado, no cabe duda que ha habido un importantísimo cambio cuantitativo, durante la última década, en lo que se refiere a la escolarización.

Otro cambio importante que han podido constatar los colegios en los últimos años ha sido como en los alumnos gitanos se reducía drásticamente el número de niños que tenían una escolarización tardía (por encima de los 6 años). En las entrevistas realizadas, se comprueba que ya es algo casi anecdótico, y que sólo el $7 \%$ de los colegios cuentan con alumnos gitanos que se hayan escolarizado en cursos posteriores a primero. Es significativo que, de esos colegios, varios se concentren en las mismas localidades, lo que de alguna forma dice también mucho sobre el trabajo que desarrollan o no los profesionales de cada ayuntamiento a la hora de trabajar este tema con las familias.

Por el contrario, se aprecia una fuerte tendencia a escolarizar a los niños desde la Educación Infantil, e incluso en muchos de los barrios que cuentan con guarderías municipales se ha visto como este servicio ha pasado a ser masivamente utilizado por las familias de esos barrios. El $80 \%$ de los colegios manifiesta que actualmente todos o la mayoría de los niños gitanos inician su escolaridad en la Educación Infantil. Este hecho permite una mejor adaptación del alumno al sistema escolar, puede comenzar de cero con el resto de los compañeros (tengamos 
en cuenta que la incorporación tardía es una de las principales causas que aducen los colegios para justificar el fracaso escolar allí donde los niños gitanos no cursan Educación Infantil) al tiempo que es una edad en donde las familias (tanto payas como gitanas) mantienen un mayor interés por la marcha del niño en la escuela, y un mayor número de contactos con su profesor o profesora.

Si bien la escolarización temprana es un hecho ya consolidado en muchos colegios y ciudades, lo cierto es que no en todos, tratándose de un proceso todavía en expansión y que en la mayoría de los lugares ha empezado a ser generalizado desde hace unos cinco años, por lo que ninguno de los cursos donde todos los niños gitanos comenzaron a la par con sus compañeros ha llegado ya a los últimos cursos de primaria. Por otra parte, es una queja muy generalizada por parte de los centros escolares el altísimo absentismo escolar que se produce en la Educación Infantil, que en muchas ocasiones convierte la escolarización a esa edad en un puro espejismo. Muchas familias siguen teniendo un excesivo sobreproteccionismo con respecto a sus hijos más pequeños, $y$, aunque puede ser sólo anecdótico, en varias ocasiones me han comentado algunas familias gitanas que no llevan a sus hijos a Educación Infantil por la negativa de los abuelos, los cuales continúan teniendo un importante peso en las decisiones relativas a la educación de sus nietos.

\section{ABSENTISMO ESCOLAR}

La cuestión del absentismo continúa siendo uno de los temas que más preocupa a la Administración (especialmente a Servicios Sociales) sobre la situación escolar de los niños gitanos. Al tiempo que es la variable que más suelen aducir los centros escolares para justificar el retraso escolar.

En este sentido, la Dirección General de Servicios Sociales de Valencia lleva a cabo un Plan de absentismo escolar gitano desde hace años, por medio del cual financia la contratación de 38 monitores de seguimiento escolar. A esto se suma el Borrador de un Decreto de Ordenación del Plan Local de Absentismo Escolar de la Comunidad Valenciana, que en estos días están estudiando conjuntamente la Consellería de Bienestar social y la Consellería de Cultura, Educación y Ciencia, el cual pretende crear distintas comisiones en cada localidad encargadas de prevenir, detectar y erradicar los distintos casos de absentismo escolar.

Hay que decir que la cuestión del absentismo suele ser abordada casi exclusivamente por los Servicios Sociales, y que algunos cole- 
gios lo expresan así directamente, quizás porque muchas veces han catalogado a los niños gitanos desde un primer momento como "niños de Servicios Sociales".

A pesar de esta preocupación, el absentismo escolar sigue siendo la cuestión que menos parece haber mejorado durante los últimos años en lo relativo a los escolares gitanos.

\subsection{Absentismo severo}

En lo que respecta al absentismo severo, un $27 \%$ de los centros tiene algún alumno gitano que apenas aparece por el colegio, si bien reconocen que este tipo de absentismo ha disminuido bastante en comparación con años anteriores. Es significativo que el absentismo severo sea mucho más pronunciado en aquellas ciudades donde se da una mayor concentración de familias gitanas, y donde la segregación espacial es un hecho que afecta a casi todas ellas. En este sentido me comentaba un monitor de seguimiento escolar: "el absentismo escolar es como la gripe, muy contagioso; si sólo falta un niño se aburre y a los tres días vuelve a aparecer por el colegio; si faltan dos, juegan a las canicas; cuando ya faltan seis, echan partidos de fútbol en la calle, y esa imagen es muy atractiva para el resto de niños que sí van a la escuela". Puede que la explicación del "contagio" influya de alguna manera en que el absentismo casi total se produzca mayoritariamente en ese contexto determinado. Curiosamente, son varios los colegios de esos barrios que comentan que tienen alumnos payos con situaciones de desestructuración familiar con igual tipo de absentismo.

\subsection{Temporeros agrícolas}

El trabajo agrícola es la principal ocupación de muchas de las familias gitanas, especialmente en los pueblos y, en ocasiones, provoca migraciones temporales a otras comarcas o provincias (el Medio Vinalopó para la uva de mesa; Requena-Utiel para la vendimia; la naranja en diferentes puntos de Valencia y Castellón; fruta en Lérida; la aceituna en Jaén; etc.). Si bien no es algo generalizado, y quizás en retroceso en muchas localidades, lo cierto es que sigue habiendo muchas familias que se desplazan durante periodos de tiempo que van de dos semanas a dos meses aproximadamente. De la misma forma, hay muchas localidades que reciben la inmigración de familias gitanas durante los periodos de recolección.

Las familias suelen llevarse a los hijos, muchos de los cuales permanecen más de un mes al año desescolarizados por este motivo, ya que son muchos los que no se escolarizan durante el tiempo que per- 
manecen en la localidad de trabajo. Los Servicios Sociales alegan que es difícil conocer el número de familias que llegan, y los niños que llevan, por lo que el tema les desborda. Por su parte, los colegios de origen, a pesar de conocer la situación año tras año, no suelen ponerse en contacto con la localidad de destino para informarles de que van a llegar esos niños, y se limitan a quejarse del retraso escolar que provocan esas ausencias tan prolongadas.

También es cierto que un número cada vez mayor de niños sí se están escolarizando durante ese tiempo, y que en distintos municipios se están empezando a dar casos de familias que dejan a los niños a cargo de un familiar (normalmente la abuela) para que no pierdan clase por los desplazamientos. A esto ayuda la existencia de comedor escolar y, aunque puede estar influido por las ayudas económicas que algunas familias reciben de Servicios Sociales, lo cierto es que se trata de un indicador de cambio.

\subsection{Absentismo esporádico}

Por otra parte, el absentismo esporádico, el que se da sólo alguna vez a la semana (por quedarse durmiendo un día por la mañana, o porque han venido unos familiares a pasar unos días, o porque tienen a alguien en el hospital, o sencillamente porque hacía mal tiempo), sigue estando muy extendido y se da de forma similar en la mayoría de los colegios, aunque con grandes diferencias dependiendo de cada familia. Más del $80 \%$ de los colegios entrevistados comenta que al menos una parte de sus alumnos gitanos tienen este tipo de absentismo. Aún así, se constata que en muchos de estos centros ha disminuido mucho el número de faltas, muchas de las cuales se concentran en los hijos de determinadas familias más desestructuradas, al tiempo que los hijos de otras familias mejor situadas sólo faltan por causas más o menos justificadas (en este sentido, influye cuál es el concepto que se utilice para considerar qué puede justificar el que los niños no vayan un día al colegio, y parece bastante claro que los criterios escolares y los familiares no acaban de coincidir). A pesar de que el absentismo escolar sea más importante en familias con menos recursos, o más desestructuradas socialmente, en varios pueblos se dan casos de familias con buenos recursos económicos (vendedores de mercado con puestos fijos, e incluso propietarios de tiendas) y muy bien integradas socialmente, cuyos hijos presentan altas tasas de absentismo escolar. La explicación podría estar en que estas familias tienen ya muy claro cuál va a ser el futuro laboral de sus hijos, y consideran que para ese trabajo ya saben bastante, a lo que se suma el que la posible presión que puedan ejercer los Servicios Sociales es mucho menor que en aquellas familias que perciben ayudas económicas. 
Los comedores escolares han supuesto en muchos colegios un importante freno al absentismo de niños gitanos, viéndose disparado dicho absentismo durante los meses de septiembre y junio en que no hay comedor. Con este servicio, los padres pueden ir a trabajar sin la preocupación de tener que llevarse a los niños, o estar en casa al mediodía para prepararles la comida. A pesar de que los colegios reconocen la importancia de este recurso, hay algunos centros (ignoro el número, pero conozco varios casos) que siguen utilizando las expulsiones del comedor como forma de castigo para los niños. Considero que ese tipo de medida en realidad a quien está castigando es a los padres, y así me lo han expresado algunas familias gitanas, a la vez que aumenta aún más la distancia existente entre colegio y familias.

\subsection{Abandono escolar prematuro}

Otra cuestión relacionada con el absentismo es el abandono escolar prematuro. Al igual que comentábamos en el apartado anterior que ha habido un cambio importante en lo relativo a que los niños tienen cada vez una escolarización más temprana, también se ha dado una mayor permanencia escolar en los cursos posteriores, aunque no tanto como en la educación infantil. Lo cierto es que, en términos generales, cada vez se abandona los estudios con mayor edad existiendo un número cada vez más significativo de niños que terminaban la antigua enseñanza obligatoria.

En varios colegios me han hecho referencia al hecho de que, en la medida en que se ha visto mermada la posibilidad de colaborar con los padres en determinados trabajos, antes de cumplir los 16 años (principalmente en trabajos agrícolas), ha ido descendiendo el abandono escolar prematuro. Mientras que otros trabajos, como la recogida de chatarra, en algunos casos la venta ambulante y, desde luego, la ayuda en las tareas del hogar por parte de las niñas, hacen que en muchas ocasiones no se llegue a terminar la escolaridad al tener que colaborar con la familia. El aumento de la escolarización temprana, incluidas las guarderías, ha supuesto paralelamente una mayor libertad para que las hermanas mayores puedan acudir con regularidad al colegio, al quedar liberadas de sus obligaciones de cuidado de los hermanos pequeños en la casa.

El 50\% de los colegios entrevistados manifiestan que la mayoría de sus alumnos gitanos abandonaban el colegio antes de los 14 años. A esto hay añadir la nueva situación creada con la implantación de la E.S.O., de la cual hablaremos después.

Los resultados académicos de los niños gitanos continúan siendo, en términos generales, más bajos que los de sus compañeros, acumu- 
lando muchos de ellos un importante desfase entre sus conocimientos y el curso en el que están. Así, en muchos colegios, las Ilamadas "clases de apoyo" se ven ocupadas mayoritariamente por niños gitanos. Por ejemplo: en un colegio de la provincia de Alicante, donde sólo hay una decena de alumnos gitanos, el maestro "de apoyo" me comentaba que en su clase únicamente estaban los niños gitanos y los niños con sindrome de Down, y que cuando enviaba a alguno de los niños gitanos a su clase, el profesor no tardaba más de 10 minutos en devolverlo a la clase "de apoyo". En cualquier caso, existen grandes diferencias dependiendo de distintos colegios, profesores, familias y niños. Así, hay un número minoritario, pero significativo, de colegios donde los niños gitanos van en general al mismo nivel que sus compañeros. Estos centros suelen coincidir con aquellos donde el porcentaje de niños gitanos es menor, aunque no en todos los casos son colegios con pocos escolares gitanos, ni en todos lo colegios donde hay pocos niños gitanos se da esa circunstancia (en algunos, todo lo contrario).

Las causas que resaltan mayoritariamente los centros escolares para explicar el alto fracaso escolar de los niños gitanos son: el absentismo escolar y la falta de interés por parte de las familias en lo referente al colegio. Sólo en dos colegios plantearon la posibilidad de que el sistema escolar tuviese también alguna responsabilidad en esa situación. Sin embargo, al hablar con las familias gitanas, éstas no parecen vivir de la misma forma el fracaso escolar, el cual puede entenderse como un concepto payo, dado que los objetivos que un importante número de las familias tienen cuando escolarizan a sus hijos quedan sobradamente cubiertos cuando abandonan el colegio.

\section{TIPOS DE COLEGIOS}

La cuestión de la doble vía escolar, colegios públicos y colegios privados, también afecta de forma significativa a la escolarización de los niños gitanos, sirviendo, en un gran número de localidades, como filtro utilizado por familias de niños payos para que sus hijos no coincidan en un mismo colegio con "los gitanos".

En lo que respecta a los colegios privados no concertados, la ausencia de niños gitanos es total, como nos dejaba bien claro la directora de uno de estos centros: al establecer el primer contacto telefónico con los colegios de la ciudad de Valencia para conocer en qué colegios habia escolares gitanos, la directora del centro respondió de malos modos " ¿es que usted no sabe dónde ha llamado? jesto es un colegio privado!, jaqui no vienen gitanos!. 
Por su parte, los colegios privados-concertados apenas cuentan con población gitana; en un altísimo porcentaje de localidades no hay ningún niño gitano en los colegios concertados. En otras, encontramos colegios con un número muy pequeño de escolares gitanos, normalmente de familias con ciertos recursos económicos que están muy sensibilizadas por el futuro escolar de sus hijos y piensan (como muchos padres payos que llevan a sus hijos a esos centros) que allí van a recibir una enseñanza de más "calidad". Existiendo también algunos casos de colegios concertados, reconozco que pocos, que sólo escolarizan a algunos niños gitanos cuando tienen problemas en algún curso para poder cubrir el mínimo de alumnos por aula. Como en todo hay excepciones, y al menos cuatro colegios concertados tienen un alto porcentaje de alumnos gitanos, lo que les ha supuesto renunciar a la mayor parte de alumnos de clases medias y altas que alguno de ellos tuvo en otro tiempo. Estos colegios tienen claro que la asistencia de niños gitanos es una mala publicidad para el centro: al recoger la información en unas de las localidades preguntamos en uno de los colegios concertados y nos dijo que no tenían ningún alumno gitano. Pero más tarde, al hablar con una de las familias gitanas nos enteramos de que sí había un alumno gitano, por lo que volvimos a ir al colegio. La directora, con mucha discreción, nos dijo que no podía hacernos la entrevista, porque nadie en el colegio sabía que iba un niño gitano y no quería que se enterasen. En cualquier caso, el hecho de que los cursos de Educación Infantil no estén concertados, y haya, por tanto, que pagar importantes mensualidades, sumado a las normalmente altas cuotas de APAS y gastos de uniforme, está sirviendo de criba para que los gitanos no accedan de forma mayoritaria a este tipo de centros, incluso en algunos casos donde, por las políticas de vivienda, la población gitana reside actualmente junto al colegio concertado.

Algo parecido, aunque de distinta forma, está sucediendo con los colegios de línea en valenciano. Estos centros son poco elegidos por las familias gitanas, las cuales no suelen hablar en valenciano, y en algunas localidades se concentran en estos colegios los hijos de familias (no gitanas) más preocupadas por los resultados escolares de sus hijos. En varios colegios donde existe línea en valenciano y línea en castellano, nos comentaban los directores, que la diferencia de resultados escolares entre ambas era grandísima, y que en la línea en castellano era donde se matriculaban todos los gitanos, así como los hijos de las familias payas más desestructuradas.

Es en los colegios públicos donde se concentra la inmensa mayoría de alumnos gitanos, especialmente en aquellos situados más cerca de los barrios de residencia de sus familias. Ya comentábamos antes el fuerte proceso de ghettización que se ha dado en muchos de estos cen- 
tros, algunos de los cuales han pasado de contar con un $40 \%$ de alumnos gitanos a tener un $90 \%$, en apenas 10 años. Este hecho, muy generalizado en los barrios más desestructurados, ha llevado en algunas ciudades al cierre del centro escolar al quedarse con un bajísimo número de niños matriculados. Pero los niños han pasado al colegio más próximo (en muchas ocasiones con ya un importante porcentaje de escolares gitanos), el cual ha visto mermado el número de matrículas de niños payos de forma inmediata. Existen algunos casos donde se han cerrado en un corto plazo de tiempo dos colegios de forma consecutiva.

$\mathrm{Al}$ igual que comentábamos en el caso de los colegios concertados, los colegios públicos también conocen la mala publicidad que les aporta el tener alumnos gitanos. Es sorprendente ver como se repite en un gran número de localidades el que haya un colegio catalogado por todo el mundo como "el colegio de los gitanos". Muchos de estos directores reconocen que muchos padres payos optan por otros colegios para que sus hijos no vayan con "los gitanos" e incluso algunos trasladan a sus hijos a otros centros. Lo curioso es que en algunos de estos colegios tan "etiquetados" sólo hay un 5\% de escolares gitanos.

Existen entre muchos colegios unas fuertes tensiones a causa del número de alumnos gitanos. En unas ocasiones por parte de la dirección del centro, que se queja que un colegio cercano no quiere tener comedor, o que apenas tiene becas de comedor, para no tener niños gitanos y que a causa de eso ellos tienen que cargar con un alto número de éstos. Y, en otras, por la presión de la asociación de padres de algunos colegios que han solicitado enérgicamente a la dirección del centro y al ayuntamiento que se ponga numeros clausus a la matrícula de niños gitanos, para que se repartan de forma equitativa por todos los colegios.

\section{PREJUICIOS Y ESTEREOTIPOS: EN LAS ANTÍPODAS DE LA INTERCULTURALIDAD}

A pesar de que en algunas ocasiones he podido leer artículos que apuntaban al País Valenciano como "vanguardia" de la interculturalidad escolar, especialmente a partir de la publicación de diversos materiales en este sentido y de la creación de la figura del asesor de educación intercultural, la dura realidad demuestra que no sólo existe una sequía casi total en este aspecto (con una o dos excepciones escolares muy concretas, y la buena voluntad a nivel individual de algunos maestros y maestras), sino que nos seguimos encontrando en las antípodas del interculturalismo. En las entrevistas realizadas, donde a priori los dis- 
tintos directores y profesores tenían una gran cautela para no emitir determinadas opiniones que pudiesen ser catalogadas de prejuiciosas o racistas, han sido innumerables las anécdotas donde lo que se reflejaba era exactamente lo contrario.

Como hemos comentado anteriormente, los niños gitanos siguen siendo vistos en un gran número de colegios como una amenaza para su reputación, y la imagen que se tiene sobre ellos y sus familias está muy marcada por los prejuicios y los estereotipos. Abundan las frases del tipo: "en general los gitanos van muy retrasados, pero hay uno que va muy bien, no parece gitano"; "algunos valen para estudiar, aunque como buenos gitanos son muy gandules"; "los padres se dedican a vender muebles viejos y fruta que robarán por ahî"; "...no sé en qué trabajan los padres, no quiero generalizar, pero los gitanos son todos unos gandules"; etc. En este sentido, es curioso que en las entrevistas abunden de forma redundante las declaraciones de directores que ante la pregunta de: ¿qué tipo de profesiones suelen desarrollar los padres de los alumnos gitanos?, den como primera contestación, y en ocasiones como única contestación, "en la venta de droga", o el que respondan "en trabajos marginales como la venta ambulante".

Las etiquetas con las que muchos colegios catalogan lo que entienden por un alumno gitano lleva en ocasiones a situaciones extremas: en un colegio de la provincia de Alicante pude conversar con varias profesoras mientras esperaba a que llegase el director. Estas no se ponían de acuerdo sobre si los niños de un par de familias determinadas eran gitanos o no. Al llegar el director me aclaró que no eran gitanos, sino unas familias inmigradas de la provincia de Jaén. Unos minutos después, mientras consultaba la lista de alumnos para ver el número de gitanos que había en el centro me dijo que a los de Jaén también los incluía, "porque esos son como los gitanos". Algo parecido, pero a la inversa, me sucedía en otro colegio: el director me comentó al comenzar la entrevista que sólo tenía 4 alumnos gitanos, pero al rato recordó que en realidad eran 6, diciendo: "se me habian olvidado los otros dos porque esos niños están muy bien integrados", pero hasta el final de la entrevista siguió hablando de lo mal que se integraban los gitanos, obviando en todo momento a los dos que estaban tan bien integrados". Es algo así como si para algunos directores y profesores la imagen de lo que es un gitano estuviese perfectamente moldeada, y lo que cuadra con ese molde es un gitano (lo sea o no lo sea) y lo que no encaja no lo es.

$\mathrm{Al}$ mismo tiempo, el desconocimiento sobre la cultura del Pueblo Gitano es muy generalizado, tanto entre los profesionales de la enseñanza como entre otros profesionales que trabajan a menudo con gitanos, como son los/as trabajadores/as sociales. Así, encontramos algu- 
nas afirmaciones de directores según los cuales: "las niñas gitanas no pueden seguir estudiando a partir de los 12 años porque lo prohibe la ley gitana". O trabajadores/as sociales que te dicen "en este pueblo antes estaba la figura del rey de los gitanos, pero ahora ya se ha perdido".

\section{LA IMPLANTACIÓN DE LA E.S.O.}

Uno de los nuevos aspectos que ha empezado a afectar a los escolares de nuestro país es la implantación de la Enseñanza Secundaria Obligatoria. Cuando se realizaron las entrevistas de este estudio apenas se había comenzado a impartir el primer curso de la ESO, por lo que la repercusión de esta nueva situación no podía todavía conocerse. En el País Valenciano, la inmensa mayoría de los colegios públicos han asumido, al menos en principio, el primer ciclo de la ESO, por lo que no ha supuesto grandes cambios en los colegios al no tener que desplazarse a otros centros. Sin embargo, el curso 98/99 que ahora comienza, va a ser el primer año para el segundo ciclo de la ESO $\left(3^{\circ}\right.$ de ESO) y va a suponer el paso de un importante número de escolares gitanos de sus antiguos colegios de EGB (ahora de primaria) a los Institutos de Enseñanza Secundaria (IES). Este es un momento histórico de transición, donde se abren nuevas expectativas de futuro para aumentar la formación de los jóvenes gitanos, pero donde también surgen nuevos peligros, por ejemplo, que al acabar $2^{\circ}$ de ESO no continúen en el IES o, lo que sería peor, que cuando los dos ciclos de ESO estén en el IES muchos escolares gitanos finalicen su escolarización en $6^{\circ}$ de Primaria. En dos de los colegios donde se había puesto en práctica la incorporación de los alumnos al IES desde $1^{\circ}$ de ESO, los directores del colegio de primaria me comentaban que los niños gitanos no habían continuado, ni siquiera aquellos que iban muy bien en sus cursos y que según sus profesores de haber continuado con el anterior sistema habrían finalizado hasta $8^{\circ}$ sin ningún problema.

Existen diferentes factores que pueden hacer peligrar lo que en principio se puede considerar una excelente medida, como es el aumentar el tiempo de formación de los alumnos gitanos:

1. Los colegios de primaria suelen estar muy cerca de los barrios de residencia de los niños gitanos, facilita el acceso y la confianza de los padres hacia la escuela. Mientras que muchos de los IES están situados a más de $1 \mathrm{~km}$. de sus casas, en ocasiones en la periferia del municipio opuesta a donde se sitúan las viviendas sociales, etc.

2. El horario en los IES es diferente, y no incluye comedor escolar, lo que puede complicar a muchas familias su situación al mediodía. 
3. Muchos padres gitanos desconfían abiertamente de unos centros que desconocen por completo, pero que perciben como un riesgo para sus hijos, y especialmente para sus hijas (van chicos más mayores, miedo a las malas compañías, al tabaco u otras drogas, etc.).

4. Hasta ahora, una parte del éxito obtenido en la escolarización temprana de niños gitanos se ha basado en la responsabilidad delegada por los padres a los hermanos mayores para que los acompañen y los vigilen en los recreos. Si los hermanos cambian de colegio a los 12 años, algunos niños pequeños pueden aumentar su absentismo, o bien que los mayores no sigan en el IES por tener que acompañar a sus hermanos al colegio. En este sentido, en un colegio de primaria que cuenta con el primer ciclo de la ESO me comentaban que una niña gitana había estado faltando a clase por la mañana porque ella salía a las 13 horas, y sus hermanos pequeños a las 12, por lo que si iba a clase no podía acompañarlos a casa.

5. Los profesores de los IES (y es hacer una generalización un tanto arriesgada) no parecen ser muy conscientes, por el momento, del cambio de alumnado que van a tener, y puede que el cambio en la forma en que reciben la enseñanza los niños, posiblemente menos cercana e individualizada, haga que muchos de ellos abandonen el IES de forma prematura.

Existe un gran escepticismo en los centros de primaria sobre la repercusión que va a tener la implantación del segundo ciclo de la ESO. Muchos de ellos se limitan a afirmar que no va a cambiar nada "porque de todas formas van a dejar el colegio cuando ellos quieran", mientras que otros muchos consideran que va a suponer un retroceso en el camino recorrido hasta ahora, especialmente si se imparte el primer ciclo de la ESO directamente en los IES. Los únicos centros que consideran que la implantación de la ESO va a ser muy positiva son aquellos donde sus alumnos gitanos terminaban desde hace ya varios cursos hasta $8^{\circ}$, y sus resultados académicos son similares a los de sus compañeros no gitanos. También lo consideran como positivo aquellos centros que cuentan con el $2^{\circ}$ ciclo de la ESO en el mismo centro (especialmente concertados) ya que el no tener que cambiar de centro aumenta las posibilidades de que los niños terminen toda la escolarización obligatoria.

Comentando esta situación con varios inspectores escolares, se mostraban también muy preocupados por el futuro de la implantación del $2^{\circ}$ ciclo de ESO, especialmente por el choque que supone el cambio de centro. Estos inspectores me sugirieron que sería interesante que en determinados centros con alto número de escolares gitanos se pudiese impartir toda la ESO, tal y como se está dando en la inmensa mayoría de colegios concertados, lo cual quizás garantizase el que un 
mayor número de niños y niñas gitanos terminasen hasta los 16 años, al tiempo que podría ser un aliciente para que algunos padres payos no cambiasen a sus hijos de colegio por el mero hecho de que van gitanos.

\section{LA ENSEÑANZA NO OBLIGATORIA}

En términos generales, tanto la enseñanza secundaria no obligatoria (bachiller y FP) como la universitaria siguen desiertas de alumnos gitanos. Apenas se encuentran 1 ó 2 jóvenes en cada una de las ciudades medias que continúen sus estudios más allá de lo que fue la EGB, mayoritariamente en FP, y con unas altas tasas de abandono en los primeros cursos. En este aspecto, la presión social del resto de familias gitanas sobre aquellos jóvenes que continúan estudiando suele ser muy fuerte. Una mujer gitana cuyo hijo cursaba $5^{\circ}$ de FP administrativo en el curso 96/97 me decía : "mis primos me dicen que si no le da lache a mi hijo, tan mayor y en la escuela, en vez de estar ayudando a sus padres en el mercado. Pero yo le animo mucho para que siga". De la misma forma, el resto de compañeros payos, muchas veces no acostumbrados a compartir colegio con ningún gitano supone también una presión sobre estos alumnos, que se encuentran en ese sentido bastante solos: un gitano que realiza estudios de FP de electricidad me comentaba que, cuando llegó por primera vez al instituto todo el mundo se le quedaba mirando y se volvían por los pasillos, escuchando comentarios del tipo "mira ese es gitano". Así, se dan casos de algunos gitanos universitarios que prefieren mantener totalmente en secreto su identidad gitana ante el miedo de ser discriminados por sus compañeros: conozco el caso de una chica que estudia magisterio en la Universidad de Alicante, la cual tiene la piel muy oscura, y para que no la discriminen por ser gitana le dice a todo el mundo que es sudamericana.

Otro importante factor que parece influir en la menor motivación por parte de las familias y los alumnos a la hora de seguir estudiando es el hecho de que de los ejemplos que conocen de gitanos que han continuado con sus estudios muchos terminan finalmente trabajando en lo mismo que sus primos que no estudiaron. Son muchos los colegios que nos dicen que no existe ninguna diferencia laboral entre los niños que abandonaron el colegio a los 12 años, que los que terminaron su escolarización con graduado escolar, o algún curso de Bachiller. Algunos profesores me comentan, con respecto a la evolución en la escolarización de los niños gitanos, que cada vez salen más preparados y muchos con el graduado, pero que acaban en la misma situación 
económica que ya tenían sus padres. Los estudios no garantizan de por sí ningún futuro y, en muchos casos, el cambio estriba en la consecución de una pobreza más ilustrada.

También puede influir la existencia de una fuerte tendencia a trabajar en lo mismo que ya trabajan los padres, como si estuviese predeterminado su futuro, por lo que el planteamiento de continuar con otros estudios puede estar fuera de ese plan de vida en muchos casos casi inevitable.

A todo esto se suma el miedo al "apayamiento" que muchas familias gitanas siguen teniendo, y la presión que sufren los jóvenes que continúan estudiando para demostrar que, a pesar de eso, siguen siendo gitanos. El director de un colegio nos contaba que mantuvo una entrevista con un padre gitano para tratar de convencerlo de que permitiese que su hijo siguiese estudiando en secundaria, a lo que el padre contestó que su hijo no podía estudiar porque ellos eran personas normales. Ante esta afirmación el director le preguntó que qué entendía por ser personas normales, contestándole el padre: "pues hacer lo que hacen todos".

Al hilo de las declaraciones de este padre, sobre la influencia de lo que hacen "todos", me parece muy interesante resaltar la existencia de al menos 3 localidades (las 3 con menos de un centenar de vecinos gitanos), donde las familias gitanas han asumido la necesidad de que los jóvenes continúen estudiando más allá de los estudios primarios, existiendo en cada localidad un alto porcentaje (en comparación con la situación general del País Valenciano) de chicos y chicas que cursan estudios de FP y Bachiller, así como un par de casos de universitarios. Otro tanto pasa en determinados linajes que se extienden por distintos pueblos, muchas de las veces con origen en la provincia de Granada, donde en distintas localidades he podido comprobar que alguna de las personas que está estudiando se encuentra emparentada con otro estudiante de otro pueblo. En este sentido, es también significativo el caso de Castellón ciudad.

A pesar de todas las dificultades existentes, el número de estudiantes gitanos que continúan con sus estudios ha aumentado mucho, si bien no al ritmo que muchos hubiéramos deseado. En el País Valenciano hay actualmente alrededor de 150 jóvenes cursando enseñanza secundaria no obligatoria, y al menos una veintena en la Universidad.

Para terminar este tema, considero que es necesario y urgente la creación de un mayor número de programas que:

1. Fomenten el conocimiento entre estudiantes gitanos que cursan enseñanzas no obligatorias (FP, Bachiller y Universidad) para evitar la soledad y el desaliento que muchos de ellos sufren. 
2. Subvencionen suficientemente esos estudios, para que nadie deje de estudiar por problemas económicos, cosa que en algunos casos está sucediendo.

3. Sirvan para dar a conocer al resto de familias gitanas la existencia en múltiples ciudades de jóvenes gitanos que estudian, propiciando así un ambiente de "normalidad" de los estudios medios y universitarios.

4. Propicien una rápida incorporación laboral tras la finalización de esos estudios, con el fin de que puedan servir como referentes positivos, exactamente lo contrario que sucede cuando el paro y los trabajos no cualificados son el resultado final de esos estudios.

\section{BIBLIOGRAFÍA}

ABAJO ALCALDE, J. E.: La escolarización de los niños gitanos. Ministerio de Trabajo y Asuntos Sociales. Madrid, 1997.

FERNÁNDEZ ENGUITA, M.: Escuela y etnicidad: el caso del Pueblo Gitano. CIDE-MEC. Madrid, 1996.

FERNÁNDEZ JIMÉNEZ, D. L.: Situación y perspectivas de la juventud gitana en Europa. Instituto Romanó. Barcelona, 1996.

GAMELLA, J. F.: La población gitana en Andalucía. Junta de Andalucía. Sevilla, 1996.

GÓMEZ ALFARO, A.: La gran redada de gitanos. Presencia Gitana. Madrid, 1993.

LEBLOND, B.: Los gitanos de España. Gedisa. Barcelona, 1987.

LIÉGEOIS, J.-P.: Gitanos e itinerantes. Presencia Gitana. Madrid, 1987.

SAN ROMÁN ESPINOSA, T.: Vecinos gitanos. Akal. Madrid 1976.

SAN ROMÁN ESPINOSA, T.: La diferència inquietant: velles $i$ noves estratègies culturals dels gitanos. Alta Fulla. Barcelona, 1994.

VV.AA.: I Congreso Gitano de la Unión Europea. Instituto Romanó de Servicios Sociales y Culturales. Barcelona, 1996.

VV.AA. del Colectivo de Enseñantes con Gitanos de Valencia : Cultura Gitana: propuestas para un trabajo intercultural en la escuela. Conselleria de Cultura, Educación y Ciencia. Valencia, 1989.

VV.AA.: La comunidad gitana en Galicia. Xunta de Galicia. Santiago de Compostela, 1991.

VV.AA.: Los gitanos en la historia y la cultura: actas de un congreso. Junta de Andalucía. Granada, 1995. 